\title{
AVL System with Smart Transmission Rate
}

\author{
Jorge Luiz Alves Trabanco, Rogério Rodrigues Amarante, Diogenes Cortijo Costa, Maria Teresa Françoso and
} Edvaldo Simões da Foseca Junior

Department of Transports, School of Civil Engineering, Architecture and Urban Design, State University of Campinas, São Paulo 13468-100, Brazil

\begin{abstract}
The use of AVL (automatic vehicle locator) systems has increased considerably. By using an AVL system, it is possible to know vehicle positions at the dispatch center, which allows the use of several applications, such as safety and security, logistics, and emergency response. High communication and data storage costs, however, lead to a low position update rate with the AVL products available, causing poor track representation, and making the route determined by the vehicle in urban areas almost illegible. This paper proposes a new approach by using intelligent techniques to choose the best position update moment to improve track representations. The principle underlying these techniques is based on vehicle status analysis (speed, direction and timing), which tries to determine when a position update is required, in order to better represent the path that a vehicle has traced, thus avoiding excessive communication and data storage. Therefore, the better the correspondence between the traced track and the real track followed by the vehicle, the greater the added value offered by system applications. This enhancement to the representation of the track allows the creation of new applications in the realm of AVL systems, particularly for situations where accuracy plays an important role.
\end{abstract}

Key words: AVL, GPS (global positioning system), dispatch, positioning.

\section{Introduction}

AVL (Automatic vehicle locator) systems basically report vehicle locations for purposes of fleet dispatch and management. AVL systems usually allow for track $\log$ storage and query, whose quality depends on several factors.

If improving the quality of AVL systems by enhancing tracking representation is an objective, the first asset to be considered is usually increasing precision of the positioning system. But this is not sufficient, as attention should also be given to the position update rules at the dispatch center.

Although AVL technology has reached a high level of development, and its utilization has seen continuous growth, the products available in Brazil are usually configured to update position periodically at low position update rates, in order to provide a good cost/benefit ratio, low data traffic, and to eliminate

Corresponding author: Jorge Luiz Alves Trabanco, $\mathrm{PhD}$, research fields: GNSS, AVL, gravimetry and geotechnology applied to transport. E-mail: jorgetrabanco@gmail.com. unnecessary data storage. This low update rate, however, causes deficiencies in track representation that a vehicle traced, mainly in urban areas, as shown in Fig. 1. On the other hand, a high update frequency (Fig. 2) means better infrastructure at the dispatch center, with increased communication and data storage costs.

This work presents solutions for track representation enhancement in order to increase vehicle adherence to the real displacement path without significant cost increment, thus avoiding excessive position update transmission.

This approach entails solutions that focus on vehicle displacement status and the selection of positions received from the GPS (global positioning system) receiver that should actually be sent to the dispatch center. Considering that one of significant AVL operating costs is related to wireless communication from vehicle to servers of dispatch center, it is mandatory that update rates are minimized without affecting tracking representation. 


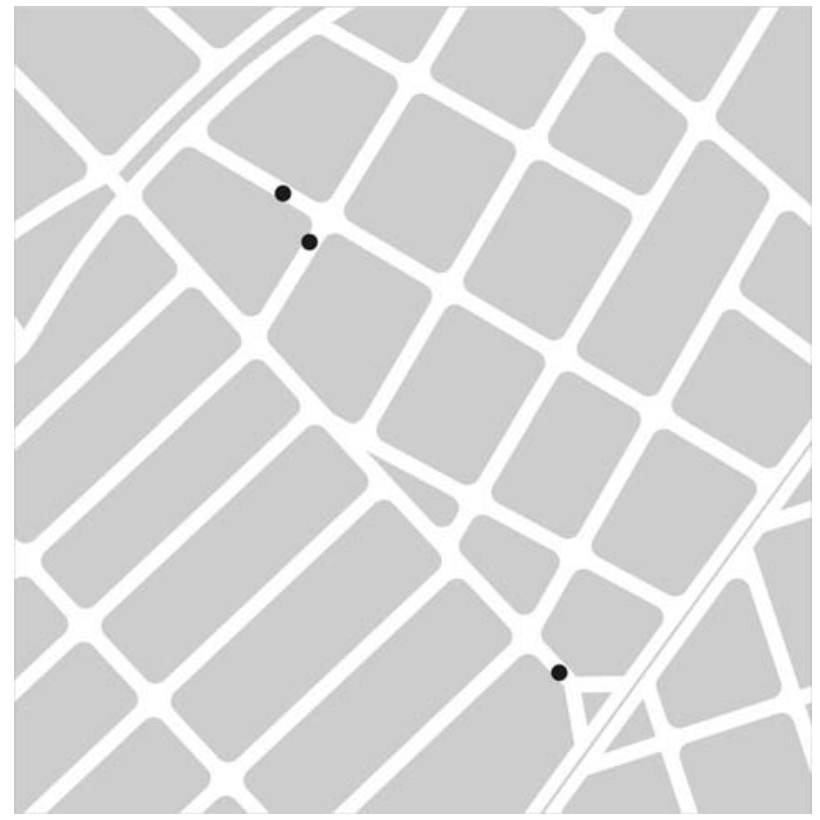

Fig. 1 Low-frequency periodic update rate.

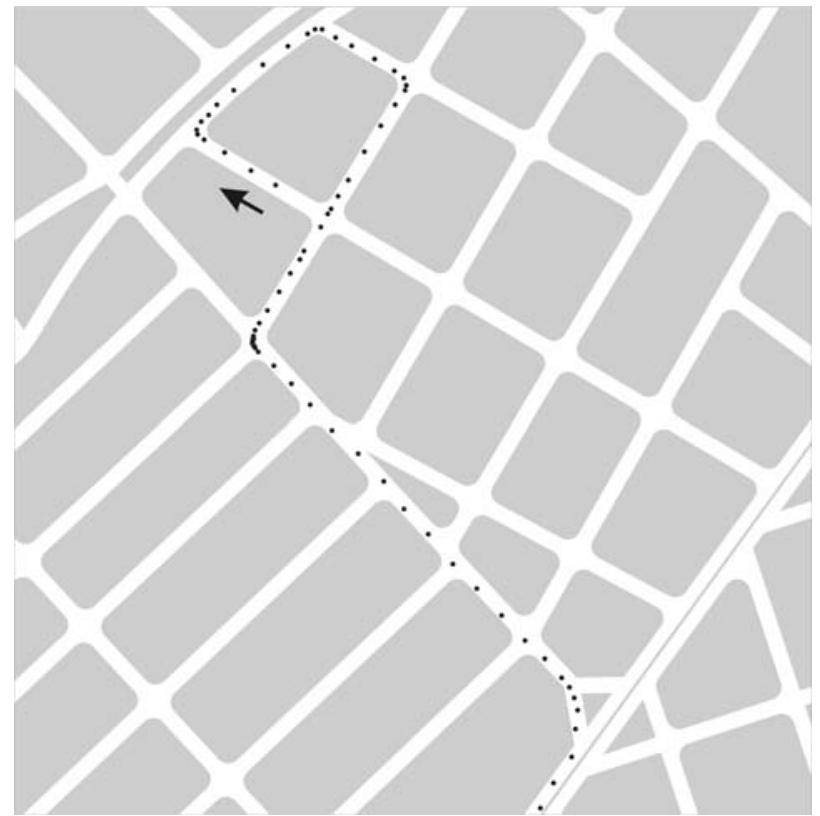

Fig. 2 High-frequency periodic update rate.

The aim of the system described herein is not to present a solution for all AVL systems, but simply to propose an approach with intelligent update rules so as to allow new applications that consider more factors than exclusively position system accuracy.

\section{A System Focused on Technology Development}

To test new approaches aimed at enhanced track representation, an AVL system was developed and deployed to allow flexibility in its development (Fig. 3). However, implementing the IPU (intelligent position update) technique presented in this paper does not depend on the development of a new system, it can also be deployed in previously developed systems.

For the mobile unit hardware assembly, a data modem board was chosen, as shown in Fig. 3, with a built-in communication system GSM/GPRS (Global System for Mobile Communications/General Packet Radio Service), a GPS-based positioning system and, for the AVL central processing unit, an ARM7 (ARM is a registered trademark of ARM Ltd., Cambridge, UK.) processor [1]. The built-in position system on the data modem board uses standard position GPS satellite through signals civilian [1].

For the AVL module operation, building complementary boards was necessary for integration of some components, including: GPS antenna, mobile phone antenna, complementary board with power supply, SIM Card socket, and serial connector.

This board could not be handmade, since the existing connector for board interfacing is very small. An SMD (superficial mounting device) silking and welding process was performed by an outsourced manufacturer.

The AVL module (shown in Fig. 4) was divided into three boards: the main board, which essentially contains the source of electric current, the interface board, which contains the SIM socket, and the circuit for serial communication voltage adjustment.

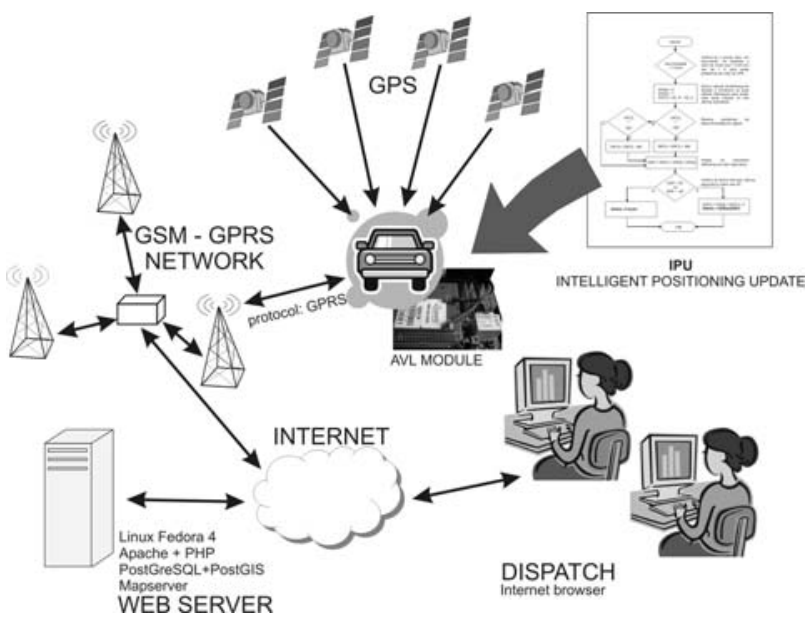

Fig. 3 AVL system assembled for IPU implementation tests. 


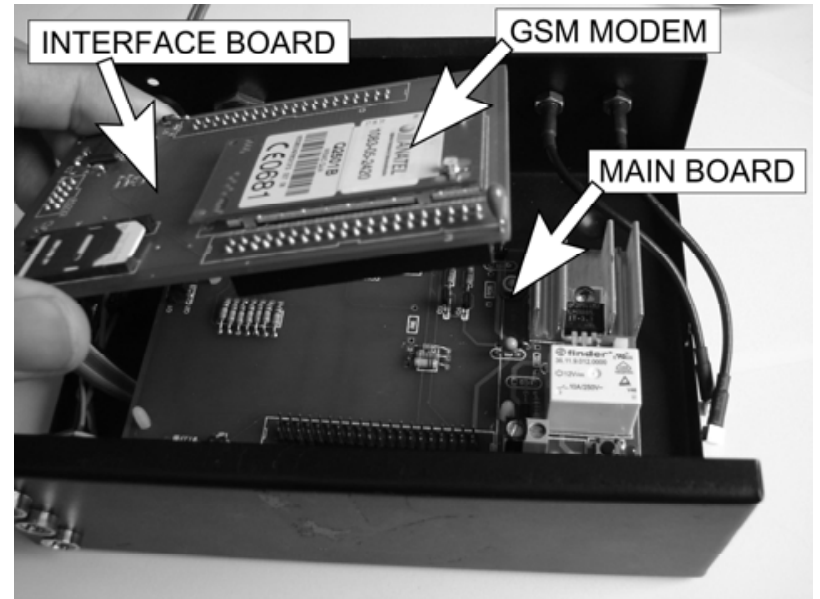

Fig. 4 AVL Module.

All boards were mounted in a metallic box that was later placed in a vehicle with coupled antennas. The installation in the vehicle was quite simple, since module only needed an electrical source. As this system is often used for security purposes, appropriate position of the components was necessary such that its deactivation can be prevented in case of robbery.

The developed module allows the coding in programming language " $\mathrm{C}$ ". The programming editor was Microsoft Visual $\mathrm{C}++6.0$ and the compilation/link was made by GCC v 3.0.4 (GNU Compiler Collection) for ARM.

The experimental dispatch system was developed based on the Internet, where configuration of a Web server was necessary. So as to make the system cheaper, preference was given to open source solutions. Among the choices are the Linux Fedora four operating system, the PostgreSQL database management system with Post GIS (geographic information system) library for geographic objects, Quantum GIS editor, Apache home page server, the Mapserver GIS Web library, the Java programming languages, the PHP hypertext preprocessor, and PL/pgSQL language. This assembly contains vector maps, and raster map images and database.

Although the mobile unit sends no update for short periods of time, which leads to a small time-lag regarding the current position of the vehicle in the dispatch center, position prediction is possible via the latest updated vectorial data containing position, direction, and speed [2-3].

\section{Systems with Fixed Position Update Rate}

The update rules currently used in AVL systems normally in use in Brazil focus on fixed rates that can be set in agreement with the application requirements. In situations where greater precision is needed, the update frequency is increased, generating unnecessary traffic and data storage. On the other hand, low update rates cause deficiencies in track representation mainly in urban areas.

In urban areas, AVL systems with a low position update rate show the track representation of the dispatched vehicle in an imprecise way, as shown in Fig. 5. The gray line represents the real track traveled by a vehicle, whereas the black dots show the vehicle with a 2-second update rate, and the black line shows tracking with a 60 -second frequency.

It is evident that conformance of tracked representation and real vehicle track through techniques based on periodic position updates requires an increased update frequency. Likewise, it is clear that obtaining good performance with no excess in data transmission and storage requires the development of a new approach toward the update method.

Several techniques have been suggested to prevent excessive vertices in route representations tracked through AVL systems. Such techniques include the use of Kalman filter and map matching [4], aperiodic Filtering [5], the use of sensors in the vehicle. Some techniques are directly deployed in the mobile unit, filtering data prior to transmission to the dispatch center, as suggested in [6], where the decision on the positioning update is based upon sensors that check brake lights, turn signals, odometer, and accelerometer. This patent-described technology suggests the utilization of a MDT (mobile data terminal) in addition to a regional map in the module incorporated in the vehicle, to help determine the updates moments [6], implementation of such a system is complex. One issue 


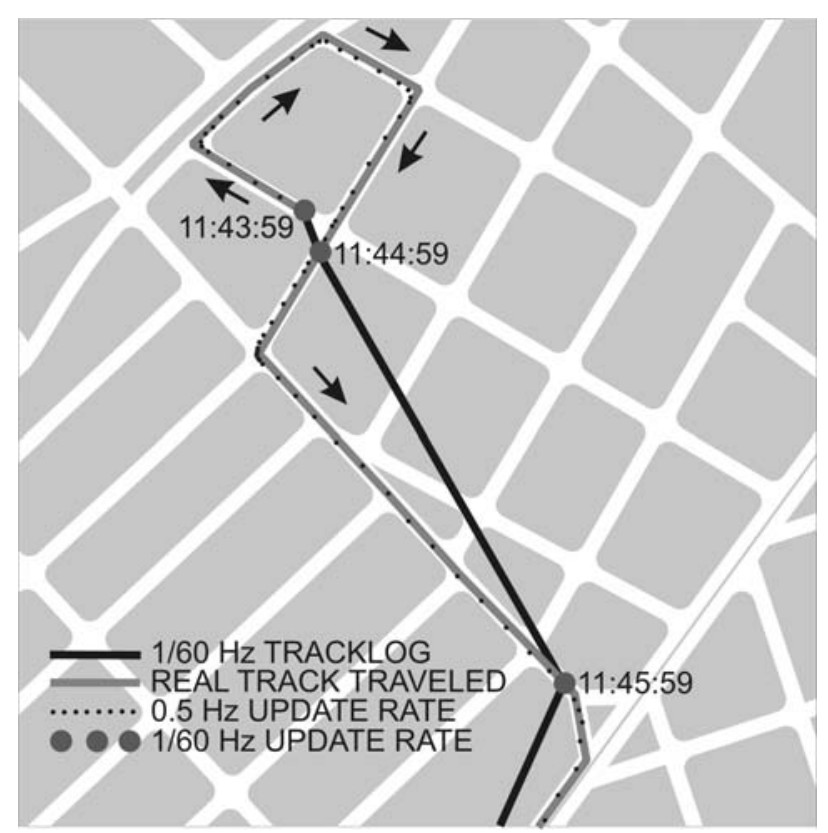

Fig. 5 Representations of various positions update rates.

that arises from map-dependent technologies (map matching) is dependence upon a regional map and its quality and update level.

\section{Development of the New Approach}

In accordance with the goals of the new approach, the ideal representation of a track by AVL system within urban areas is one containing the smallest number of position updates while still adequately representing the streets, avenues, and roads traversed by a tracked vehicle, as shown in Fig. 6.

Update rate adjustment is an alternative for improving track representation, as shown in Fig. 5. However, situations such as different speed levels and sudden changes of direction can affect track representation. Table 1 compares the "ideal model" (Fig. 6) in two tested situations (update rates of $2 \mathrm{~s}$ and $60 \mathrm{~s})$.

It is important to stress that data transmission is not only one of the costs involved in AVL systems, but this factor can significantly interfere with results if not properly taken into account. However, a strong tendency toward cost reduction is apparent, due to competition among mobile phone service providers.

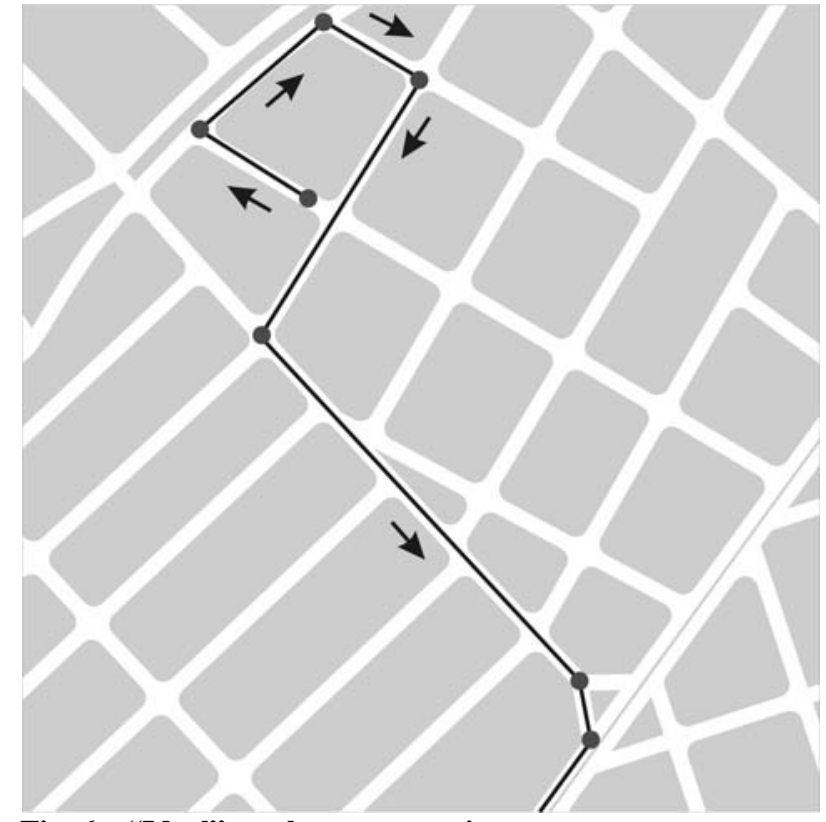

Fig. 6 “Ideal” track representation.

Table 1 Position updates.

\begin{tabular}{|l|l|}
\hline Method & updates \\
\hline update rate 2 seconds (Fig. 2) & 71 \\
\hline update rate 60 seconds (Fig. 1) & 3 \\
\hline "Ideal” (Fig. 6) & 7 \\
\hline
\end{tabular}

4.1 First Approach - Variation of Frequency According to Speed

Consider that a stationary vehicle tracked every 10 seconds generates 17,280 updates every 48 hours. Since all positions are redundant, it is not necessary to update the position at a rate of 10 seconds. It is important to mention that in a GPS receiver compatible with NMEA (National Marine Electronics Association) 0183 [7], it is possible to extract, besides position, the direction, speed, dilution of the precision and date and time. This information forms the basis for whether data must be sent or not. Thus, it was possible to develop an algorithm to be implemented in the AVL unit that triggers different update rates as a function of the speed indicated by GPS data.

Through this approach, it is possible to significantly reduce data traffic, mainly avoiding updates when the vehicle is motionless. However, it is important to note that the total absence of updates for motionless vehicles 
would not be acceptable. This is because AVL systems are used not only for position dispatch [8], but also for vehicle status verification, security, system logistics, functioning.

Although simple and functional, this approach strays from the main target of this work, that is, to approach the ideal track representation using the minimum number of nodes (updates).

\subsection{Final Approach - IPU}

In the "ideal" track representation, the existence of vertices in significant changes of direction can be observed, as shown in Fig. 6. In order to allow the implementation of technics to approach the "ideal track log", solutions must be shaped based on information and rules that go beyond the periodic update rate; these solutions together form IPU [9].

\subsubsection{Integration of the Differences in Direction}

NMEA protocol allows verification of azimuth (angle in relation to the true North) of the direction toward which the object is moving. This information permits calculation of the difference in direction at any moment, which allows identification of the moment when the direction difference becomes evident, as shown in Fig. 7. The direction change can stand for a value of $-180^{\circ}$ up to $180^{\circ}$. Negative values represent deflections to the left and positive values to the right.

Fig. 7 shows a map containing a fragment of a traced track represented by a gray line with a 2 -second GPS update rate (every update represented by a black dot). The white dots represent the moment when the direction difference function surpasses a given angle according to Fig. 8.

\subsubsection{Detection Updates Moment in Curves That} Approach to a Straight

After IPU program codification and installation on the AVL module, it was possible to verify that the track representations algorithm shows good adherence to the real tracks of the vehicle. However, there remained problems during slight changes of direction. This problem is shown in Fig. 9.
It became clear that considering only significant direction changes is not sufficient. The previously demonstrated solution needed to be refined. An approach was developed to determine update moment on long curves with large radiuses. This approach attempts to use information received from the GPS to eliminate the need to install sensors, which would increase module unit complexity and cost such that it would become unfeasible for commercial use.

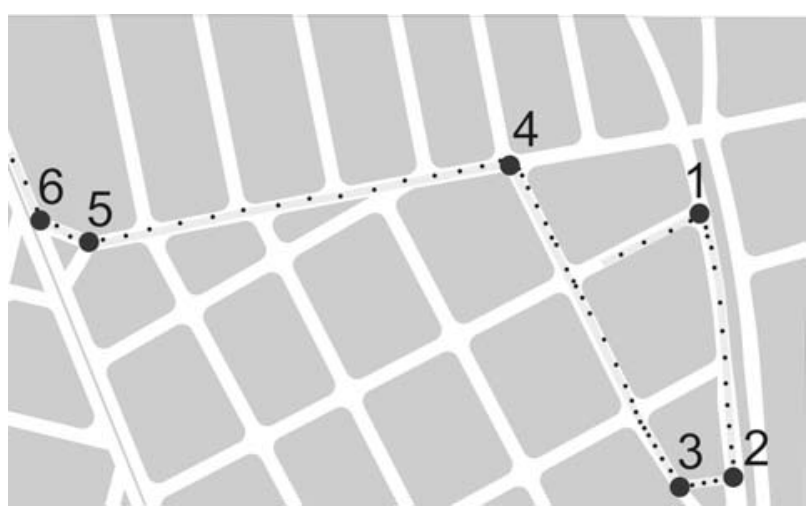

Fig. 7 Moments at which direction changes are detected.

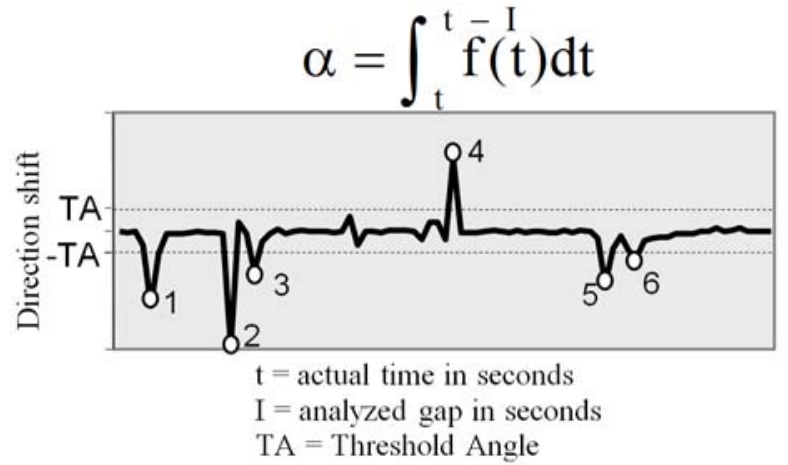

Fig. 8 Function to detect update moment.

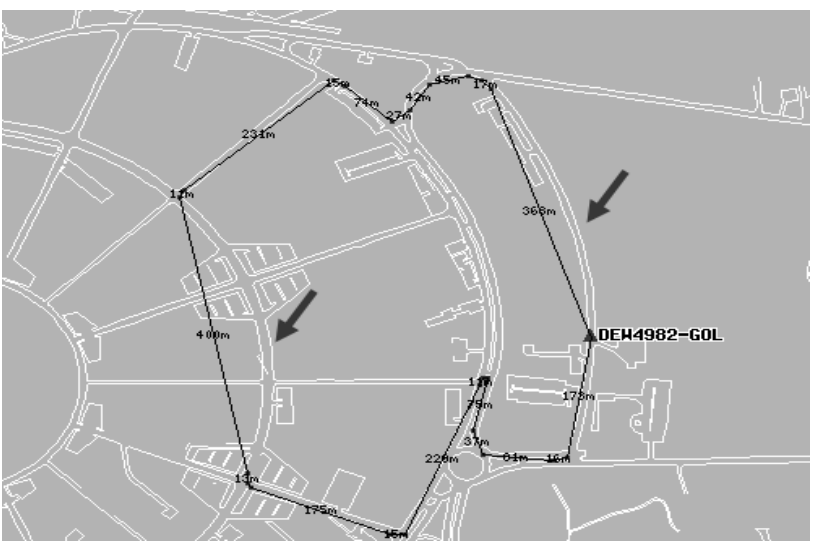

Fig. 9 Problem found when detecting a slight change of direction. 
The adopted solution sends a new update only when the estimated error of a projected vector exceeds a predetermined value, as shown in Fig. 10.

In order to estimate the exact moment when this vector line error exceeds the maximum value, it is necessary to store both the position and direction of the previously updated point. The distance of the current position to the previously updated point is calculated every time a GPS reading is performed. This distance allows calculation of the maximum angle, to be compared with the sum of the difference of direction in the interval of the previous updated point to the present moment,

$$
\text { MAX_ANG }=\arctan (\text { max_error/distance }) \text {. }
$$

In this case the value chosen for this maximum error is 40 meters, equivalent to approximately half of the standard city blocks dimension in the State of São Paulo. Note that the purpose of this algorithm is not only to increase track representation, but also to prevent excessive updating.

The calculation of the distance between the previous updated position and the current one is made through Eq. (1) to present an approximate result aimed at speed processing. This calculated distance is used only to take decisions on the best moment to update and does not affect position system accuracy.

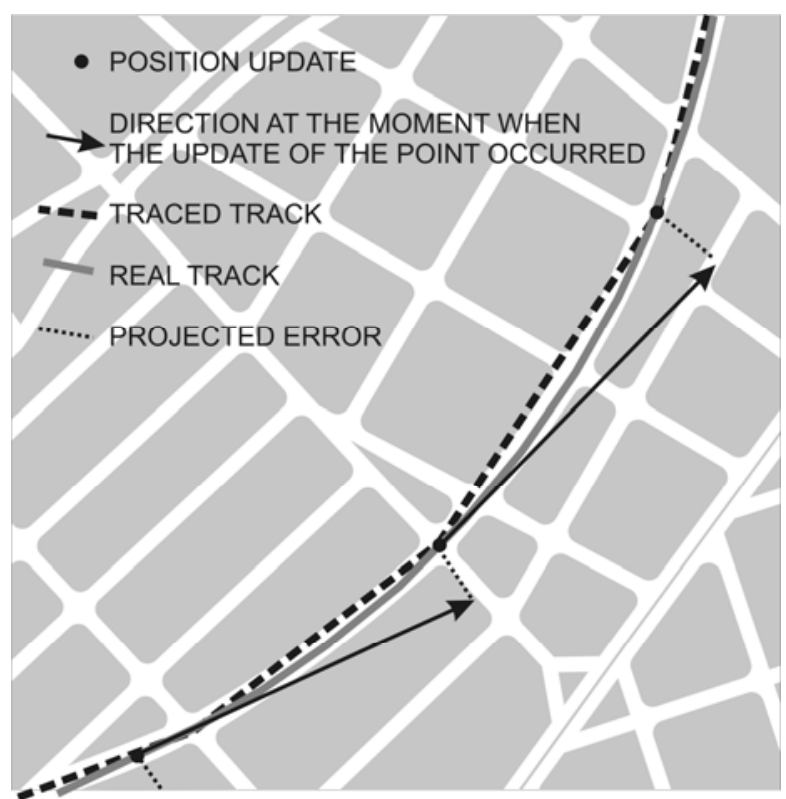

Fig. 10 Solution for wide curves with great radiuses.

$$
D I S T=\frac{40,030 \cdot \alpha}{360} \cdot 1,000
$$

where,

$$
\begin{aligned}
& \alpha=a \cos \left(\begin{array}{l}
\cos \left(\operatorname{arc} \_\varphi^{\prime}\right) \cdot \cos \left(\operatorname{arc} \_\varphi\right) \\
+\sin \left(\operatorname{arc} \_\varphi^{\prime}\right) \\
\cdot \sin \left(\operatorname{arc} \_\varphi\right) \cdot \cos \left(\operatorname{arc} \__{\_} \lambda\right)
\end{array}\right) \\
& \operatorname{arc}_{-} \varphi^{\prime}=90-\varphi^{\prime} \\
& \text { arc_ } \varphi=90-\varphi \\
& \text { arc_ } \lambda=\lambda^{\prime}-\lambda
\end{aligned}
$$

with

$\alpha$ - arc between current and last position

$\varphi$ - current latitude (LAT)

$\lambda$ - current longitude (LON)

$\varphi^{\prime}-$ last latitude updated (LAST_LAT)

$\lambda^{\prime}-$ last longitude updated (LAST_LON)

$40,030-2 \cdot \pi \cdot($ radius earth $)$

1,000 - from kilometers to meters

This formula leads to the creation of a function that returns the distance to be used in the slight-curve detection algorithm. The result of the combination of the two techniques is set forth in Fig. 11.

\subsection{Modeled Example Algorithm}

Although the mathematical modeling of the problem was developed for a continuous world (integrals and derivatives), the algorithms were implemented for a discrete world (sums and differences).

The flowchart example proposed in Fig. 12 must run after each GPS data reading. When the value of the "UPDATE" variable is "TRUE", the position must be updated at the dispatch center. The point indicated as "A" in the flowchart illustrates begin of part of the algorithm that detects moments of deflection, and point "B" indicate the start of piece of the algorithm that detects the moment updates on smooth curves.

To test how the algorithm works in addition to fine tuning constants TA (threshold angle) and max error used at the most appropriate times for vertex update, within urban areas a tracking frequency of one-second was evaluated. Taking the collected mass of data as a starting point, the algorithm was tested several times, 


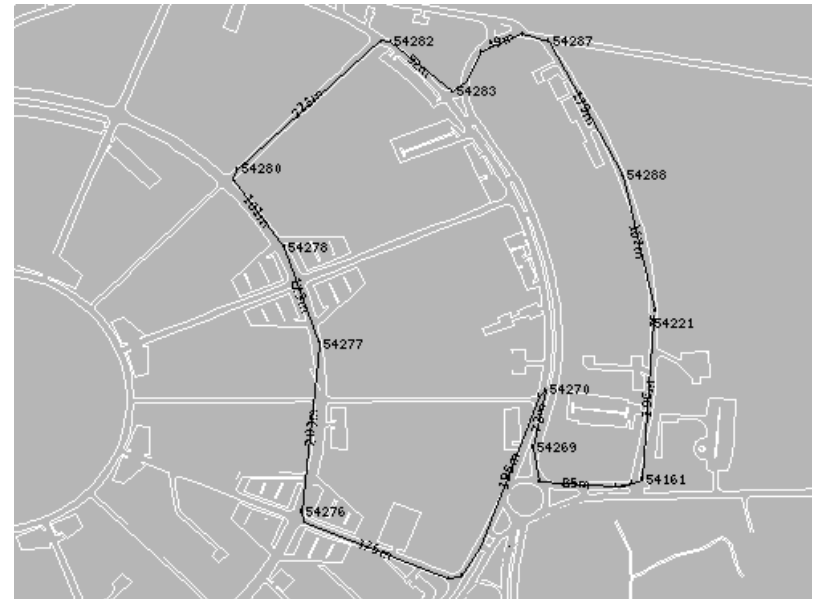

Fig. 11 Result of combination of both solutions.

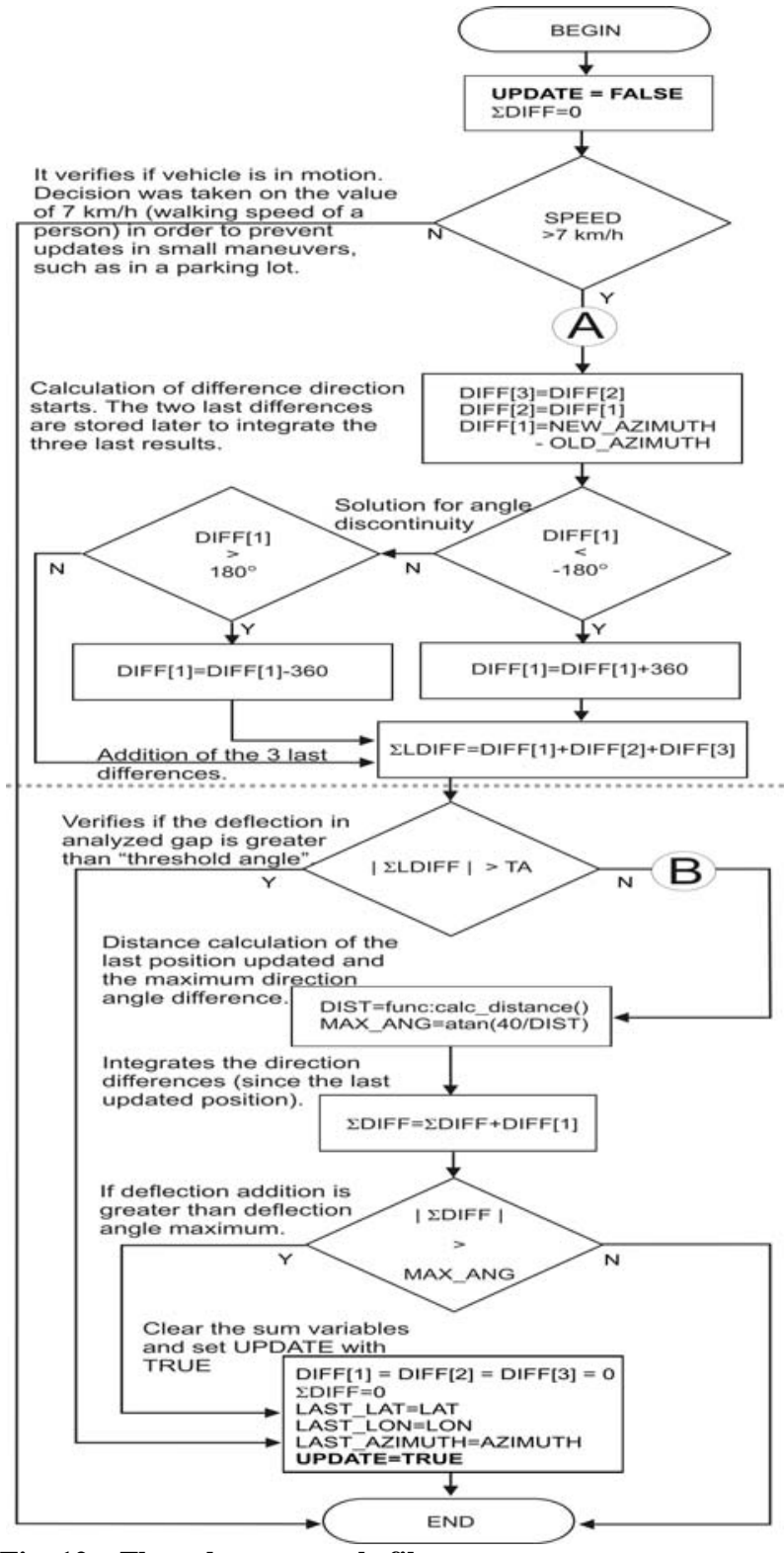

Fig. 12 Flow chart example filter. each time, the value of constants was varied up to the point where the smallest possible number of vertices was detected without affecting the quality of representation. Fig. 13 shows a simulation of the algorithm on a path composed with different types of curve, and the updates moments represented by dots. Fig. 14 shows the proportion of positions that were filtered or detected by algorithms A and B.

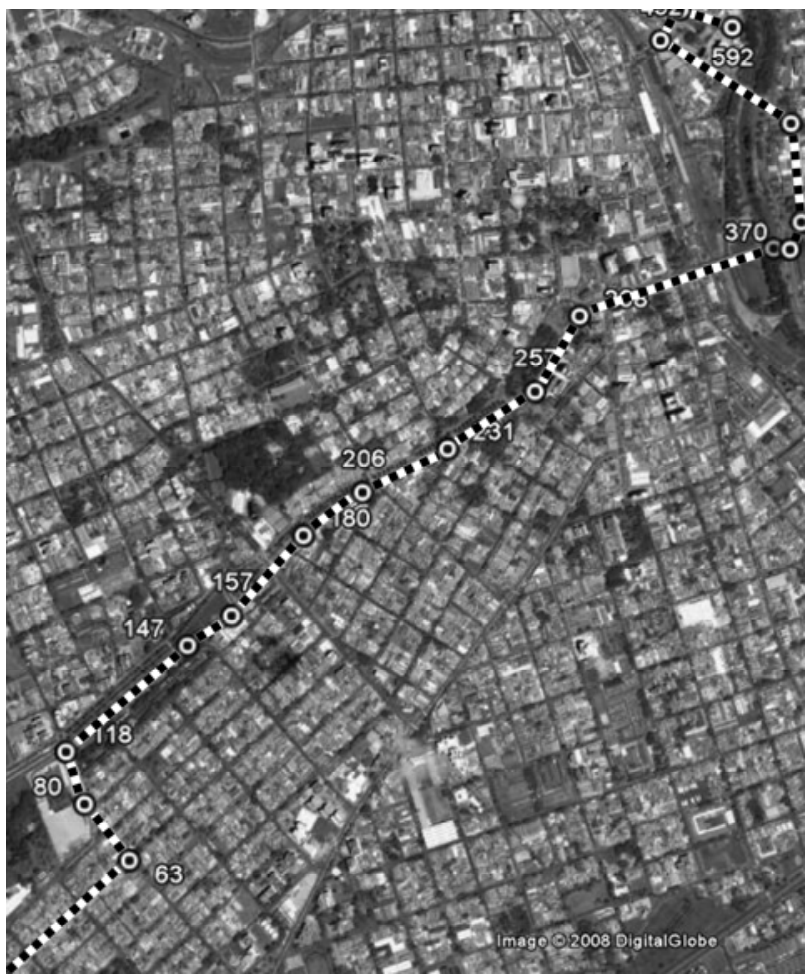

Fig. 13 Example of a tested track.

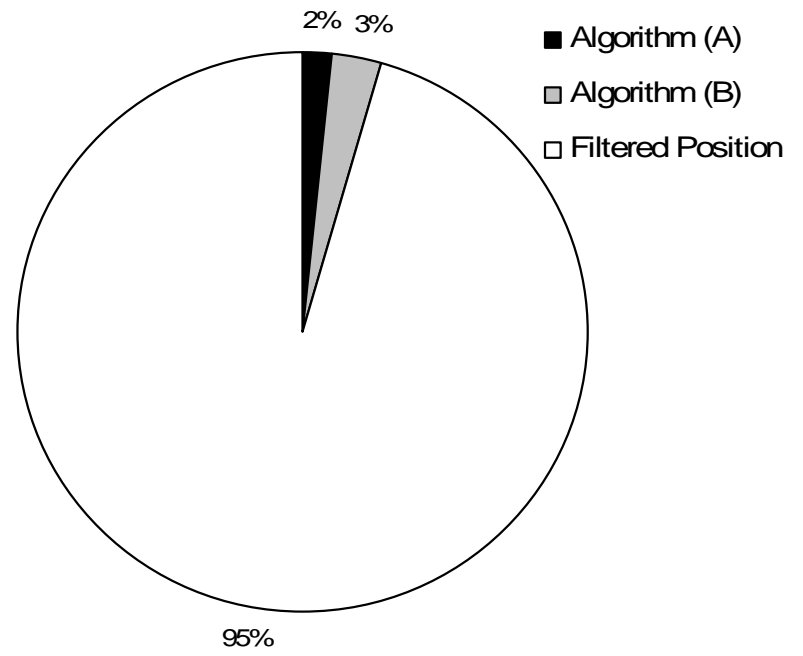

Fig. 14 Position raw data with $1 \mathrm{~Hz}$. 
The simulation data show that $41^{\circ}$ for constant $T A$ and $50 \mathrm{~m}$ for max_error were optimal values such that the algorithm presented a positive result for the surroundings of those points predefined as ideal for maximum quality of representation. It is notable that $23 \%$ of the updated values can be considered interference, since they do not affect the representation. Modify the unknowns TA \& max_error to reducing the number of updates beyond such values, in case as show in Fig. 13, would negatively affect representation. It is possible that different results may be obtained in regions presenting different features. Therefore, it is necessary to use a safety margin or to test performance in each region where the system is to be deployed.

To avoid erroneous interpretation of data absence (it could be understood by the dispatch center that the mobile unit was defective), it is necessary to send the AVL mobile unit status to the dispatch center within a specified time period, which may vary according to the application (e.g., 15 minutes).

If the communication system stops working, it is necessary to store the position data in the AVL module memory, and, when possible, to send the data automatically.

Even though each string contains only a few bytes, transmission packet size is bigger, as it contains other information concerning the GPRS/IP/TCP/HTTP protocols. Based on this information, the presented algorithm must be modified, where the data position is stored in a queue, as needed, followed by transmitting and emptying the queue content within a predetermined frequency.

The purpose of this work is not to present an optimized algorithm for AVL system position updates; ours is only one suggestion amongst many possible combinations. However, it is clear that an intelligent approach based on tracked object displacement is needed to obtain better track representation without excessive position updates.

\subsection{Operation}

The developed system was tested over four months by three mobile units. During this period, many details needed to be adjusted so as to improve quality, debug errors, and to increase system reliability. Two of the mobile systems were installed in company vehicles, while the third was installed in a private car. The main characteristic of commercial usage is intense utilization only during working hours, while a private car is typically used in more random circumstances, including weekends.

Accordingly, two measurements are notable:

The instrumented commercial vehicles showed approximately 750 position updates per day, during working time and virtually zero updates during the weekend.

The instrumented private car showed an average of 400 updates per day, with great variation from day to day.

The quantity of updates is not equivalent to the number of data packet transmissions; therefore, some updates are made at several different moments. Even if this fact is not considered, the amount of data transmission averages approximately 8.46 megabytes per month, according with the sample of the four vehicles tested, which, in comparison with a standard system (with 1-minute preset update rate -20.27 $\mathrm{MB} /$ month) would offer as much as a $58 \%$ savings on data transfer. However, it is quite difficult to precisely measure the exact savings, since mobile phone companies sell the service of data packets in a wide range of plans, e.g., minimum package of data and

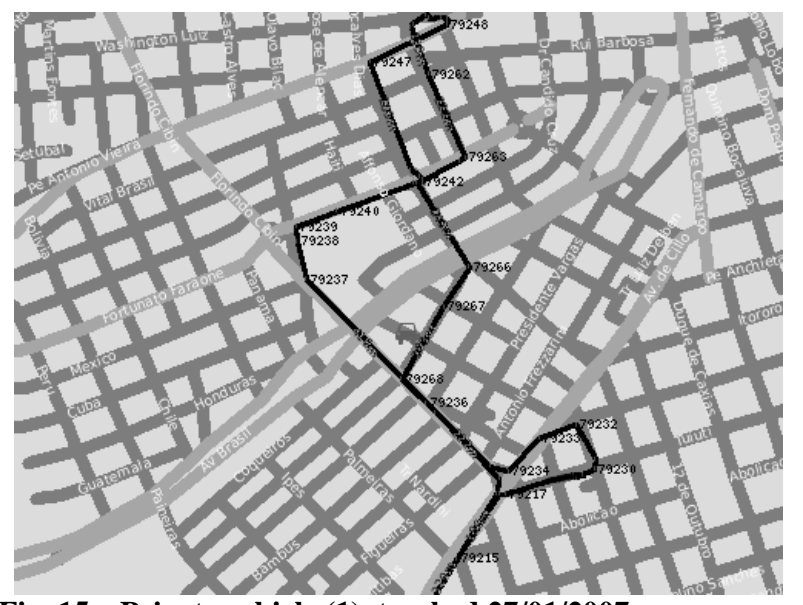

Fig. 15 Private vehicle (1), tracked 27/01/2007. 
costs for decreasing megabytes as a function of traffic volume and information technology (IT) infrastructure.

Through IPU, it is possible to accurately represent the ways in which a vehicle tracked, as shown in Figs. $15-18$.

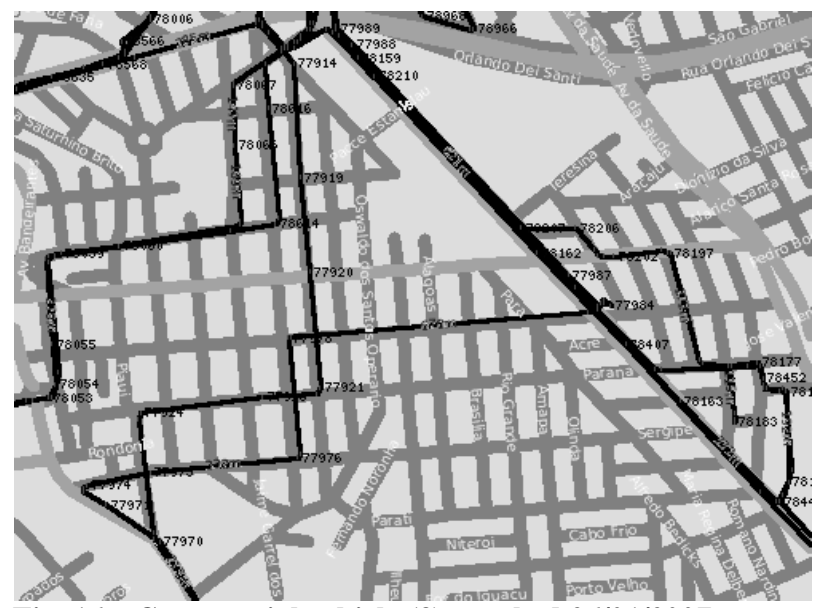

Fig. 16 Commercial vehicle (3), tracked 26/01/2007.

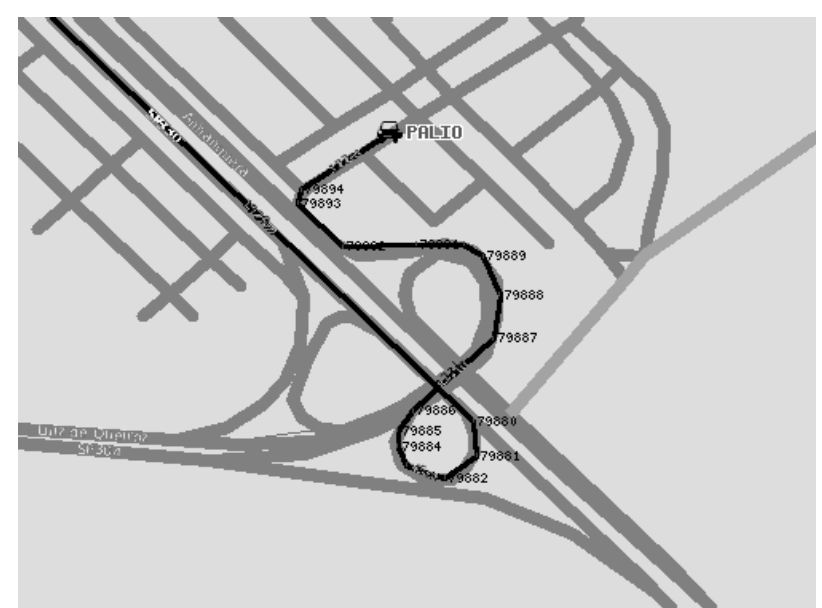

Fig. 17 Commercial vehicle (3), tracked 29/01/2007.

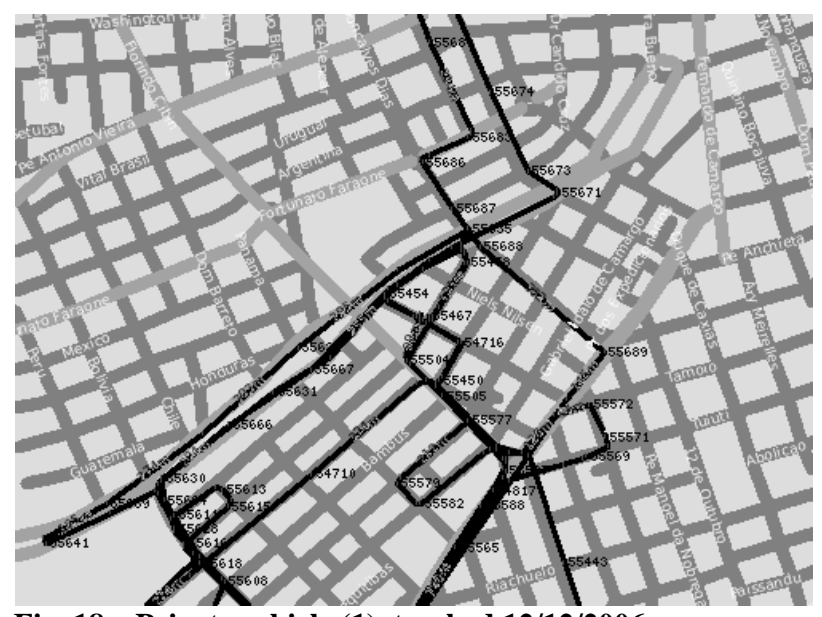

Fig. 18 Private vehicle (1), tracked 12/12/2006.

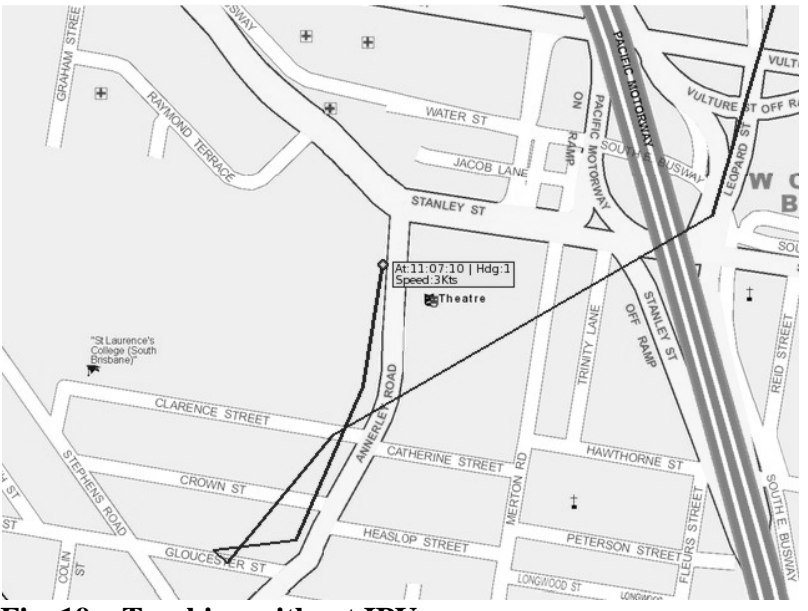

Fig. 19 Tracking without IPU.

Source: January 20, 2007, http://www.traceme.tv/Demo.

As a point of comparison, Fig. 19 shows a tracked vehicle without IPU.

\section{Conclusions}

This work develops an approach to improve track representation using a new methodology that considers the ideal moment for position updating. Although adherence between track representation and real vehicle track was improved, there was no significant increase in the number of position updates, avoiding increased costs with respect to communication and data storage. The importance of this issue is the fact that communications systems represent a significant component operating costs. Because despite of the tariffs plans data of mobile phone getting cheaper, AVL systems are used to monitor many vehicles which become necessary investment on IT infrastructure of central monitoring in order to avoid gateway congestion. If compared with a standard preset 1-minute update rate, the proposed system after above mentioned in small numbers of testing can or maybe can offer reduction on data traffic volume.

More precise track representation facilitates the creation of new AVL applications that need more accuracy, like detecting areas where the map is still incomplete and allowing track analysis made by fleet drivers for optimization and orientation. Improved track representation may also lead to improved 
preventive maintenance on fleet vehicles as a function of better understanding of how mileage is accumulated in each vehicle.

\section{References}

[1] R. R. Amarante, AVL system development with intelligent position update rules that enhance tracks representations, Master's Thesis, State University of Campinas, 2007.

[2] A. Karbassi and M. Barth, Vehicle route prediction and time of arrival estimation techniques for improved transportation system management, in: Intelligent Vehicles Symposium, Proceedings of the IEEE, June 9-11, 2003, pp. 511-516.

[3] N. Papadoglou and E. Stipidis, Investigation for a global AVL system, IEEE Transactions on Intelligent Transportation Systems 2 (3) (2001)121-126.

[4] E. J. Krakiwsky, C. B. Harris and R. V. C. Wong, A Kalman filter for integrating dead reckoning, map matching and GPS positioning. Navigation into the $21 \mathrm{st}$ Century, in: IEEE Position Location and Navigation Symposium, Nov. 29-Dec. 2, 1988, pp. 39-46.
[5] L. Nguyen and M. Barth, Improving automatic vehicle location efficiency through aperiodic filtering, in: Transportation Systems Conference, Toronto, Canada, Sep. 17-20, 2006, pp. 281-286.

[6] J. T. Corwith, Automatic vehicle location system and method with reduced bandwidth requirement, United States Patent, US6236357 B1, 2001.

[7] NMEA (National Marine Electronics Association), NMEA 0183 interface standard V3.01, available online at: http://www.nmea.org/pub/0183/index.html (accessed Mar. 20, 2006).

[8] D. Gillen, D. Johnson, N. Schrank and E. Sullivan, Assessment of AVL for San Luis Obispo Transit (revised on: 2005), Department of Civil Engineering, Institute for Transportation Studies, University of California, Berkeley, available online at: http://www.wlu.ca//docsnpubs_detail.php?grp_id=748\&d oc_id $=4289$.

[9] R. R. Amarante and J. A. L. Trabanco, IPU to improve tracking representation of the AVL systems, INPI patent request, Brazilian Patent, PI0802516-9, 2008. 